\title{
A conditional stability estimate for determining a cavity in an elastic material
}

\author{
By Nobuyuki Higashimori \\ Graduate School of Informatics, Kyoto University, Yoshida-Honmachi, Sakyo-ku, Kyoto 606-8501 \\ (Communicated by Shigefumi Mori, M. J. A., Feb. 12, 2002)
}

\begin{abstract}
We consider an inverse problem of identifying an unknown cavity within an elastic material by a single boundary measurement. For this problem we show a conditional stability estimate.
\end{abstract}

Key words: Inverse problem; Lamé system; conditional stability.

1. Introduction. The purpose of the present paper is to announce a conditional stability estimate for an inverse problem of elastostatic measurement which we state in the following.

Let $\Omega$ be a cube in $\mathbf{R}^{3}$ given by

$$
\Omega=\left\{x \in \mathbf{R}^{3}:\left|x_{i}\right|<H(i=1,2,3)\right\}
$$

for a positive number $H$, and let $D$ be a subdomain of $\Omega$ such that $\bar{D} \subset \Omega$, where $\bar{D}$ means the closure of $D$. We assume that the domain $D$ is starshaped with respect to the origin of $\mathbf{R}^{3}$, and we regard the domain $\Omega \backslash \bar{D}$ as a reference configuration of a homogeneous and isotropic elastic material in natural state, the Lamé constants of which are $\lambda$ and $\mu$. We also regard the domain $D$ as a cavity which is unknown in our inverse problem. Let $\boldsymbol{u}(x)=\left(u^{(1)}(x), u^{(2)}(x), u^{(3)}(x)\right)^{T}$ denote the displacement vector at a point $x$ of the material, and assume that no surface force is applied on $\partial D$.

Our inverse problem is the identification of the unknown cavity $D$ by observing the displacement and the surface traction over an open part of the boundary of the cube $\Omega$. For this problem we show a modulus of continuity of the mapping from the observed data to the cavity in a certain class of regularity.

There have been various results on the conditional stability for inverse problems by electrostatic measurements (see e.g. [1] and its references). They reduce the problems to Cauchy problems of a single elliptic equation in order to determine unknown subdomains. We have generalized some of the results to the case of a system of elliptic equations and

2000 Mathematics Subject Classification. Primary 35R30; Secondary 35E99, 74B05. have succeeded in applying a method for electrostatic measurement to elastostatic measurement.

The details will be published elsewhere.

2. The main result. We prepare some notation and hypotheses to state our result. The main result is Theorem 1 .

For a point $x_{0} \in \mathbf{R}^{3}$ and a number $r>0$, we set $B\left(x_{0}, r\right)=\left\{x \in \mathbf{R}^{3}:\left|x-x_{0}\right|<r\right\}$, where $|\cdot|$ denotes the Euclidean norm. We use the capital $B$ to denote the unit ball $B(0,1)$. For two sets $P$ and $Q \subset \mathbf{R}^{3}, P \Subset Q$ means $\bar{P} \subset Q$. For a $\mathbf{R}^{3}$-valued function $\boldsymbol{u}, \nabla \boldsymbol{u}$ denotes the Jacobian matrix of $\boldsymbol{u}$. Define the linearized strain tensor $\boldsymbol{e}(\boldsymbol{u})$ of $\boldsymbol{u}$ by

$$
\boldsymbol{e}(\boldsymbol{u}):=\frac{1}{2}\left(\nabla \boldsymbol{u}+\nabla \boldsymbol{u}^{T}\right)=\frac{1}{2}\left(\partial_{i} u^{(j)}+\partial_{j} u^{(i)}\right)_{i, j},
$$

where $\partial_{i}=\partial / \partial x_{i}(i=1,2,3)$. Let $\lambda$ and $\mu$ be two real numbers and define the stress tensor $\boldsymbol{\sigma}(\boldsymbol{u})$ and the Lamé system $L$ by

$$
\begin{aligned}
& \boldsymbol{\sigma}(\boldsymbol{u}):=\lambda(\operatorname{div} \boldsymbol{u}) I+2 \mu \boldsymbol{e}(\boldsymbol{u}), \\
& L:=\mu \Delta I+(\lambda+\mu) \operatorname{grad} \operatorname{div},
\end{aligned}
$$

where $I$ denotes the identity matrix.

Let $\Omega$ be the cube given by (1). Suppose that one of the six faces of the cube, say $\partial \Omega \cap\left\{x_{3}=-H\right\}$, contains an open disk, which is denoted by $\Gamma$ with its radius $R_{\Gamma}$. Suppose the Lamé constants $\lambda$ and $\mu$ satisfy

$$
\mu>0 \text { and } 2 \mu+3 \lambda>0
$$

and the operator $L$ defined by (3) is strongly elliptic.

For $j=1,2$, let $D_{j}$ be a subdomain of $\Omega$ given in the form

$$
D_{j}=\left\{x \in \mathbf{R}^{3}: x \neq 0,|x|<\rho_{j}\left(\frac{x}{|x|}\right)\right\} \cup\{0\},
$$


where the function $\rho_{j} \in C^{1}(\bar{B})$ is supposed to satisfy (5) $\rho_{0} \leqslant \rho_{j}(x) \leqslant \rho^{0}(x \in \partial B), \quad\left\|\nabla \rho_{j}\right\|_{C^{0}(\partial B)} \leqslant R$ for some fixed numbers $R>0$ and $0<\rho_{0}<\rho^{0}<H$.

We set $\Omega_{j}:=\Omega \backslash \overline{D_{j}}$ and assume that functions $\boldsymbol{u}_{j} \in C^{3}\left(\overline{\Omega_{j}}: \mathbf{R}^{3}\right)$ satisfies, for $j=1,2$,

(6) $L \boldsymbol{u}_{j}=0$ in $\Omega_{j}, \quad \boldsymbol{\sigma}\left(\boldsymbol{u}_{j}\right) n=0$ on $\partial D_{j}$,

where $n$ denotes the outward unit normal. We suppose moreover that $\boldsymbol{u}_{j}$ satisfies, for $j=1,2$,

$$
\begin{gathered}
\left\|\boldsymbol{u}_{j}\right\|_{C^{3}\left(\overline{\Omega_{j}}\right)} \leqslant E, \\
\left|\operatorname{div} \boldsymbol{u}_{j}\right| \geqslant m \text { on } \Gamma,
\end{gathered}
$$

for some fixed numbers $E>0$ and $m>0$. The pair $\left(D_{j}, \boldsymbol{u}_{j}\right)$ is said to satisfy the "a priori assumption" when $D_{j}$ and $\boldsymbol{u}_{j}$ satisfy the hypotheses above.

Our main result is as follows:

Theorem 1. There exist numbers $K>0$ and $0<\varepsilon_{0}<e^{-e}$, depending only on $E, H, m, R, R_{\Gamma}, \lambda$, $\mu, \rho_{0}$ and $\rho^{0}$, such that the following holds: Suppose that two pairs $\left(D_{1}, \boldsymbol{u}_{1}\right)$ and $\left(D_{2}, \boldsymbol{u}_{2}\right)$ satisfy the a priori assumption, and that the Cauchy data $\left(\boldsymbol{f}_{j}, \boldsymbol{g}_{j}\right)$ of $\boldsymbol{u}_{j}$ is given on $\Gamma(j=1,2)$, i.e.,

$$
\boldsymbol{u}_{j}=\boldsymbol{f}_{j}, \quad \boldsymbol{\sigma}\left(\boldsymbol{u}_{j}\right) n=\boldsymbol{g}_{j} \text { on } \Gamma .
$$

Then we have

$$
\left\|\rho_{1}-\rho_{2}\right\|_{C^{0}(\partial B)} \leqslant K(\ln \ln |\ln \varepsilon|)^{-1}
$$

for $\varepsilon:=\left\|\boldsymbol{f}_{1}-\boldsymbol{f}_{2}\right\|_{L^{\infty}(\Gamma)}+\left\|\boldsymbol{g}_{1}-\boldsymbol{g}_{2}\right\|_{L^{\infty}(\Gamma)}<\varepsilon_{0}$.

3. Lemmas. In this section we give three lemmas, which are keys for our proof of the main result. These lemmas lead us to a proof of the theorem by an idea similar to that proposed in [1].

Let $\boldsymbol{u}=\left(u^{(1)}, u^{(2)}, u^{(3)}\right)^{T}$ satisfy the Lamé system $L \boldsymbol{u}=0$ in a domain $G$ of $\mathbf{R}^{3}$, and define, for $\boldsymbol{u}$, a $\mathbf{R}^{4}$-valued function $\boldsymbol{U}=\left(U_{1}, U_{2}, U_{3}, U_{4}\right)^{T}$ by $U_{i}:=$ $u^{(i)}(i=1,2,3)$ and $U_{4}:=\operatorname{div} \boldsymbol{u}$. Then $\boldsymbol{U}$ satisfies a system of equations

$$
\text { (9) }\left\{\begin{array}{rl}
\mu \Delta U_{i}+(\lambda+\mu) \partial_{i} U_{4} & =0 \\
(\lambda+2 \mu) \Delta U_{4} & =0
\end{array} \quad(i=1,2,3),\right.
$$

in the domain $G$.

We firstly give a conditional stability of the Cauchy problem for the Lamé system, which can be obtained by a Carleman estimate (see [3]) for the system (9).

Lemma 1.1. Assume that the domain $G$ has a boundary $\partial G$ which contains an open part $\gamma$ of the plane $\left\{x_{3}=0\right\}$. Let $\boldsymbol{u} \in C^{3}\left(G \cup \bar{\gamma}: \mathbf{R}^{3}\right)$ be a solution to the Cauchy problem

$$
\begin{gathered}
L \boldsymbol{u}=0 \text { in } G, \\
\boldsymbol{u}=\boldsymbol{f}, \boldsymbol{\sigma}(\boldsymbol{u}) n=\boldsymbol{g} \text { on } \gamma .
\end{gathered}
$$

Let $\omega_{1}$ and $\omega_{2}$ be two subdomains of $G$ such that $\omega_{1} \Subset \omega_{2} \Subset G \cup \bar{\gamma}$. Then there exist numbers $K>0$ and $0<\tau<1$, depending on $\lambda, \mu, \omega_{1}, \omega_{2}, \gamma$ and $\|\boldsymbol{u}\|_{C^{3}\left(\overline{\omega_{2}}\right)}$, such that we have

$$
\|\boldsymbol{u}\|_{H^{1}\left(\omega_{1}\right)}+\|\operatorname{div} \boldsymbol{u}\|_{H^{1}\left(\omega_{1}\right)} \leqslant K \eta^{\tau}
$$

for $\eta:=\|\boldsymbol{f}\|_{L^{\infty}(\gamma)}+\|\boldsymbol{g}\|_{L^{\infty}(\gamma)} \leqslant 1$.

In the next lemma, part (b) stands for a generalization of the three balls theorem for the harmonic functions (cf. [4]).

Lemma 1.2. Take a point $x_{0} \in G$ and three numbers $r>0, \beta>1, \gamma>1$ such that $B\left(x_{0}, \beta \gamma r\right) \Subset$ $G$ and $\beta \gamma r \leqslant 1$. Then we have the following:

(a) Let $v \in C^{2}(G: \mathbf{R})$ be a harmonic function on $G$. Then we have, for $\tau_{0}=\ln \gamma / \ln \beta \gamma$,

$$
\|v\|_{L^{2}\left(B\left(x_{0}, \beta r\right)\right)} \leqslant\|v\|_{L^{2}\left(B\left(x_{0}, r\right)\right)}^{\tau_{0}}\|v\|_{L^{2}\left(B\left(x_{0}, \beta \gamma r\right)\right)}^{1-\tau_{0}} .
$$

(b) Let $\boldsymbol{u} \in C^{2}\left(G: \mathbf{R}^{4}\right)$ satisfy $L \boldsymbol{u}=0$ in $G$ and let $\boldsymbol{U}$ be defined above. Then there exist numbers $K>0$ and $0<\tau<1$, depending only on $\beta, \gamma, \lambda$ and $\mu$, such that

$$
\|\boldsymbol{U}\|_{L^{2}\left(B\left(x_{0}, \beta r\right)\right)} \leqslant K\|\boldsymbol{U}\|_{L^{2}\left(B\left(x_{0}, r\right)\right)}^{\tau}\|\boldsymbol{U}\|_{L^{2}\left(B\left(x_{0}, \beta \gamma r\right)\right)}^{1-\tau} .
$$

Finally, we give an estimate for the sup-norm of the gradient $\nabla \boldsymbol{U}$ in terms of the $L^{2}$-norm of $\boldsymbol{U}$. We are led to the estimate by Schauder and local boundedness estimates for solutions to elliptic equations (e.g. [5]).

Lemma 1.3. Let $\boldsymbol{U} \in C^{2}\left(G: \mathbf{R}^{4}\right)$ satisfy (9) in $G$ and take a point $x_{0} \in G$ and numbers $0<r_{1}<$ $r_{2} \leqslant 1$ such that $B\left(x_{0}, r_{2}\right) \Subset G$. Then there exists a positive number $K$ depending on $\lambda$ and $\mu$ such that

$$
\|\nabla \boldsymbol{U}\|_{L^{\infty}\left(B\left(x_{0}, r_{1}\right)\right)} \leqslant \frac{K\|\boldsymbol{U}\|_{L^{2}\left(B\left(x_{0}, r_{2}\right)\right)}}{\left(r_{2}-r_{1}\right)^{7 / 2}} .
$$

4. A sketch of a proof for the main result. In this section the capital letters $K_{1}, K_{2}, \ldots, K_{10}$ represent numbers depending only on the parameters fixed in $\S 2$.

Set $\Omega_{12}:=\Omega_{1} \cap \Omega_{2}=\Omega \backslash\left(\overline{D_{1}} \cup \overline{D_{2}}\right)$, and assume $D_{1} \neq D_{2}$. Without loss of generality we further assume that

$$
\rho_{12}:=\left\|\rho_{1}-\rho_{2}\right\|_{L^{\infty}(\partial B)}=\rho_{2}\left(y_{0}\right)-\rho_{1}\left(y_{0}\right)>0
$$

for some $y_{0} \in \partial B$.

We observe that the domains $D_{j}, \Omega_{j}$ and $\Omega_{12}$ satisfy a cone property stated below. For $x \in \mathbf{R}^{3} \backslash\{0\}$ 
and $0<\theta<\pi / 2$, let $C_{\theta}^{ \pm}(x)$ denote a cone defined by

$$
C_{\theta}^{ \pm}(x)=\left\{y \in \mathbf{R}^{3}: \pm x \cdot(y-x)>|x||y-x| \cos \theta\right\},
$$

and let $H(x)$ be the half space

$$
H(x)=\left\{y \in \mathbf{R}^{3}: x \cdot y \geqslant 0\right\} .
$$

Here $\cdot$ denotes the inner product in $\mathbf{R}^{3}$. Then the cone property is as follows: there exists a number $0<\theta<\pi / 2$, depending only on $\rho_{0}, \rho^{0}$ and $R$ in (5), such that

(10) $C_{\theta}^{+}(x) \cap \Omega \subset \Omega_{j} \quad$ and $\quad C_{\theta}^{-}(x) \cap H(x) \subset D_{j}$

hold for all $x \in \partial D_{j}$ and $j=1,2$. Using the same value of $\theta$, we have

$$
C_{\theta}^{+}(x) \cap \Omega \subset \Omega_{12}
$$

for all $x \in \partial \Omega_{12} \cap \partial D_{j}, j=1,2$.

From Green's formula (e.g. [6]) and (6), we have

$$
\begin{aligned}
& \int_{\Omega_{1} \backslash \Omega_{12}}\left\{\lambda\left(\operatorname{div} \boldsymbol{u}_{1}\right)^{2}+2 \mu \boldsymbol{e}\left(\boldsymbol{u}_{1}\right): \boldsymbol{e}\left(\boldsymbol{u}_{1}\right)\right\} d x \\
& \leqslant \operatorname{area}\left(\partial D_{2}\right) \cdot \sup _{\Omega_{1}}\left|\boldsymbol{u}_{1}\right| \cdot \sup _{\partial \Omega_{12} \cap \partial D_{2}}\left|\sigma\left(\boldsymbol{u}_{1}\right) n\right|,
\end{aligned}
$$

where $A: B$ denotes the sum $\sum_{i, j=1}^{3} a_{i j} b_{i j}$ for two matrices $A=\left(a_{i j}\right)$ and $B=\left(b_{i j}\right)$. By setting $\boldsymbol{u}:=$ $\boldsymbol{u}_{1}-\boldsymbol{u}_{2}$ on $\Omega_{12}$, we obtain

(13) $\int_{\Omega_{1} \backslash \Omega_{12}}\left(\operatorname{div} \boldsymbol{u}_{1}\right)^{2} d x \leqslant K_{1}\|\sigma(\boldsymbol{u}) n\|_{L^{\infty}\left(\partial \Omega_{12}\right)}$

for some number $K_{1}>0$. We remark that we use (4), (12) and the a priori assumption. For sufficiently small $\varepsilon$, we can derive an estimate

$$
\|\nabla \boldsymbol{u}\|_{L^{\infty}\left(\partial \Omega_{12}\right)} \leqslant K_{2}|\ln \varepsilon|^{-K_{3}}
$$

for some numbers $K_{2}>0$ and $0<K_{3}<1$. Here we use Lemma 1.1 - Lemma 1.3, (7) and (11) (cf. [1]).

Consider the domain

$$
G:=\left\{x \in \Omega_{12}: \operatorname{dist}(x, \Gamma)<\frac{m}{4 E}, x_{3}<-\rho^{0}\right\},
$$

and note that $G$ contains a ball whose radius can be estimated in terms of $E, m$ and $H-\rho^{0}$. Then, by (10) and (11), there exists a number $K_{4}>0$ such that $B(y, 4 \rho) \subset \Omega_{1} \backslash \Omega_{12}$ and $B(z, 4 \rho) \subset G$ for

$$
\begin{gathered}
\rho:=K_{4} \min \left\{1, \rho_{12}\right\}, \\
y:=\frac{\rho_{1}\left(y_{0}\right) y_{0}+\rho_{2}\left(y_{0}\right) y_{0}}{2},
\end{gathered}
$$

and for some $z \in G$.

Since $\operatorname{div} \boldsymbol{u}_{1}$ is harmonic in $\Omega_{1}$, repeated use of Lemma 1.2 (cf. [2]) yields

$$
\text { (15) } \begin{aligned}
& \int_{B(z, \rho)}\left(\operatorname{div} \boldsymbol{u}_{1}\right)^{2} d x \\
& \leqslant K_{5}\left(\int_{B\left(y_{0}, \rho\right)}\left(\operatorname{div} \boldsymbol{u}_{1}\right)^{2} d x\right)^{\exp \left(-K_{6} / \rho+K_{7}\right)}
\end{aligned}
$$

for some numbers $K_{5}, K_{6}>0$ and $K_{7} \in \mathbf{R}$. Since we have (13)-(15) and since $|\operatorname{div} \boldsymbol{u}| \geqslant m / 2$ in $G$ by (7) and (8), we reach

$$
\rho^{3} \leqslant K_{8}\left(K_{9}|\ln \epsilon|^{-K_{3}}\right)^{\exp \left(-K_{6} / \rho+K_{7}\right)}
$$

for some numbers $K_{8}, K_{9}>0$. If $\varepsilon$ is sufficiently small, the inequality above means

$$
\rho \leqslant K_{10}(\ln \ln |\ln \varepsilon|)^{-1}
$$

for some $K_{10}>0$. Thus we obtain Theorem 1 by an appropriate choice of the number $\varepsilon_{0}$.

\section{References}

[ 1 ] Alessandrini, G., Beretta, E., Rosset, E., and Vessella, S.: Optimal stability for inverse elliptic boundary value problem with unknown boundaries. Ann. Scuola Norm. Sup. Pisa Cl. Sci. (4), 29(4), 755-806 (2000).

[ 2 ] Beretta, E., and Vessella, S.: Stable determination of boundaries from Cauchy data. SIAM J. Math. Anal., 30, 220-232 (1998).

[ 3 ] Isakov, V.: Inverse Problems for Partial Differential Equations. Appl. Math. Sci., vol. 127, Springer, New York (1998).

[4] Kukavika, I.: Quantitative uniqueness for secondorder elliptic operators. Duke Math. J., 91, 225240 (1998).

[ 5 ] Gilbarg, D., and Trudinger, N. S.: Elliptic Partial Differential Equations of Second Order. 3rd ed., Springer, Berlin (1983).

[ 6 ] Ciarlet, P. G.: Three Dimensional Elasticity. North-Holland, Amsterdam (1988). 